\title{
The predictivity of preliminary embryo-fetal development (EFD) studies: results of a retrospective survey in Japanese pharmaceutical companies
}

\author{
Noriko Okahashi ${ }^{1,2}$, Takashi Ikeda ${ }^{1,3}$, Shuichi Kai ${ }^{1,4}$, Shinichi Komatsu',5, Hajime Matsui 1,6, \\ Yasuhiro Yamashita1,7, Keiji Yamamoto ${ }^{1,8}$ and Fumio Sagami ${ }^{1,9}$ \\ ${ }^{1}$ Non-clinical Evaluation Subcommittee, Drug Evaluation Committee, Japan Pharmaceutical Manufactures \\ Association, 3-4-1 Nihonbashi Honcho, Chuo-ku, Tokyo 103-0023, Japan \\ ${ }^{2}$ Dainippon Sumitomo Pharma Co., Ltd., 33-94 Enoki-cho, Suita, Osaka 564-0053, Japan \\ ${ }^{3}$ ASKA Pharmaceutical Co., Ltd., 1604 Shimosakunobe, Takatsu-ku, Kawasaki, Kanagawa 213-8522, Japan \\ ${ }^{4}$ Bristol-Myers K.K., 5-1 Nishi-Shinjuku 6-chome, Shinjuku-ku, Tokyo 163-1328, Japan \\ ${ }^{5}$ GlaxoSmithKline K. K., 6-15 Sendagaya 4-chome, Shibuya-ku, Tokyo 151-8566, Japan \\ ${ }^{6}$ The Chemo-Sero-Therapeutic Research Institute, Kyokushi, Kikuchi, Kumamoto 869-1298, Japan \\ ${ }^{7}$ Nippon Shinyaku Co., Ltd., 14 Nishinosho-monguchi-cho, Kisshoin, Minami-ku, Kyoto 601-8550, Japan \\ ${ }^{8}$ Takeda Pharmaceutical Co., Ltd., 17-85 Jusohonmachi 2-chome, Yodogawa-ku, Osaka 532-8686, Japan \\ ${ }^{9}$ Eisai Co., Ltd., 1-3 Tokodai 5-chome, Tsukuba-shi, Ibaraki 300-2635, Japan
}

(Received September 15, 2009; Accepted October 19, 2009)

\begin{abstract}
To explore the predictivity of dose range-finding (DRF) studies, we conducted a survey by sending out questionnaires to 72 Japanese pharmaceutical companies. The survey yielded data for 108 and 85 compounds for which any embryo-fetal development (EFD) toxicities were observed in the definitive studies in rodents and non-rodents, respectively. As a result of the analysis, $83 \%$ of studies in rodents and $80 \%$ in non-rodents showed EFD effects in the DRF studies. When focusing on teratogenicity, $91 \%$ of studies in rodents and $100 \%$ in non-rodents were judged "positive" in the DRF studies when all EFD toxicities were used as markers. When the effects of both the rodent and non-rodent studies were evaluated together, the combination predictive value in the DRF studies was $96 \%$ for EFD toxicants and $100 \%$ for teratogens. To evaluate the influence of the examination items, the predictive value was analyzed using 54 compounds for which full examinations (external, visceral and skeletal examination) were conducted in both rodent and non-rodent DRF studies. When the results were judged by including or excluding skeletal and visceral examinations results, the predictive values were not significantly different. In conclusion, the results of this survey showed that a pair of the DRF studies in the rodents and non-rodents is useful to increase the predictivity of DRF studies. In addition the inclusion of observations such as fetal survival, body weight and external examination into the DRF studies are important to predict effects in the definitive studies.
\end{abstract}

Key words: EFD toxicity studies, Predictivity, Preliminary or DRF studies, Survey

\section{INTRODUCTION}

In drug development, the need to avoid the risk to the embryo or fetus is imperative when including women of childbearing potential (WOCBP) in clinical trials. To achieve this objective, use of highly effective methods of birth control or pregnancy test before and during the clinical study is essential. In addition to taking precau- tions to prevent pregnancy during clinical trials, conducting embryo-fetal development (EFD) studies can be useful to characterize the inherent risk of a compound so as to be able to better inform of the risks if an exposure during pregnancy occurs, and to make an assessment of the suitability of the compound as a development candidate. The International Conference on Harmonization of Technical Requirements for Registration of Pharmaceuticals

Correspondence: Noriko Okahashi (E-mail: noriko-okahashi@ds-pharma.co.jp) 
for Human Use (ICH) has published ICH-M3 guideline (Non-Clinical Safety Studies for the Conduct of Human Clinical Trials for Pharmaceuticals) which addresses the principles for the development of non-clinical strategies and on the timing of toxicity studies in relation to the conduct of clinical trials. In M3 (R1) guideline, there had been regional differences in the timing of the EFD toxicity studies to support the inclusion of WOCBP in clinical trials, that is, EFD studies should be completed prior to the inclusion of WOCBP in any type of clinical trial in Japan and the EU, whereas, in the US, WOCBP may be included in early, carefully monitored studies without EFD studies (ICH harmonized tripartite guideline, 2000).

EFD studies are time-consuming and require technical expertise and large number of animals. The methods and endpoints for EFD studies are stated in the ICH-S5 (R2) guideline (Detection of toxicity to reproduction for medicinal products \& toxicity to male fertility). In accordance with this guideline, the definitive EFD studies should be conducted with about 20 dams per group in 2 species (rodents and non-rodents) in compliance with good laboratory practice (GLP). From the point of view of appropriate animal usage in research, studies should be conducted at a time that is necessary to support clinical safety. However, one could question the need for such studies early in clinical trials where pregnancy is extremely unlikely while on clinical study. In contrast, the pregnancy rate in the clinical studies might be increased as clinical trials get longer and larger and less daily oversight is likely to occur. Considering these points together the balance between the scales of the clinical studies (duration of the treatment and number of the volunteers) and the sizes of the non-clinical studies are most important.

Generally, many pharmaceutical companies conduct preliminary or dose range-finding (DRF) studies before the definitive EFD studies. The DRF studies are conducted in the early stages of the drug development on a small scale and with limited endpoints. The results from the DRF studies have not been utilized for the evaluation of EFD toxicities, since the purpose was to set the dose for the definitive studies. However, they do routinely use pregnant animals. The DRF studies may vary between the companies and/or compounds because DRF studies are not regulated by the guideline. Moreover, the DRF methods, endpoints and/or predictivity for EFD effects are unknown because the details of the DRF studies have not been published.

To explore the possibility of using the information from DRF studies, we conducted a survey about the EFD studies done in the pharmaceutical companies which were members of the Japan Pharmaceutical Manufactures
Association (JPMA) in order to investigate the predictivity of the DRF studies.

\section{MATERIALS AND METHODS}

Questionnaires were distributed to 72 member pharmaceutical companies of the JPMA, and 24 responses were received (response rate $33 \%$ ). The first survey was conducted during April 6-20, 2007 and the follow-up survey was conducted during October 16, 2007- January 15, 2008.

The questionnaires were provided by the ICH M3 expert working group to obtain a set of summaries of the results of preliminary or DRF and definitive EFD studies, using a formatted answer sheet including questions on the study design (fetal examination items and the number of the litters per group) and the maternal/fetal effects (effective dose levels and the types of fetal effects) (Fig. 1). The data set was sought for compounds which met all the following criteria: (1) an EFD study had been conducted within the past 10 years, (2) both the DRF and definitive studies had been performed, and (3) any EFD toxicities were observed in the definitive studies. The "any EFD toxicities" were defined as effects such as embryo-fetal death, decreased fetal body weight, fetal malformation or variation (external, visceral and/or skeletal) and/or fetal retardation of ossification. The therapeutic areas were not limited. The data sets, consisting of data from the preliminary or DRF study and the definitive study, were obtained for 108 studies for rodents ( 5 in mice and 103 in rats) and 85 studies for non-rodents (rabbits). The net number of the analyzed compounds was 136 since 57 studies among 193 studies (108 in rodents and 85 in non-rodents) were obtained from the same compounds. The numbers of the compounds for each company are shown in Table 1.

Persons in each company who were knowledgeable about reproductive and developmental toxicity were requested to respond. The answer sheets were returned to the JPMA staff who replaced company names with code numbers to blind the analysis that was then conducted by the authors. The follow-up survey for the compounds in which teratogenic effects were not detected in the DRF studies was done to clarify the details using a interview with each company.

The data were analyzed using the identical code assigned by the allocated company number, compound number and test species. The end points of the analysis were as follows; (1) study designs of the preliminary or DRF studies, (2) predictive value of the preliminary or DRF study to detect any of the embryo-fetal toxicities in each rodent or non-rodent definitive study, (3) predic- 
The predictivity of preliminary embryo-fetal development studies

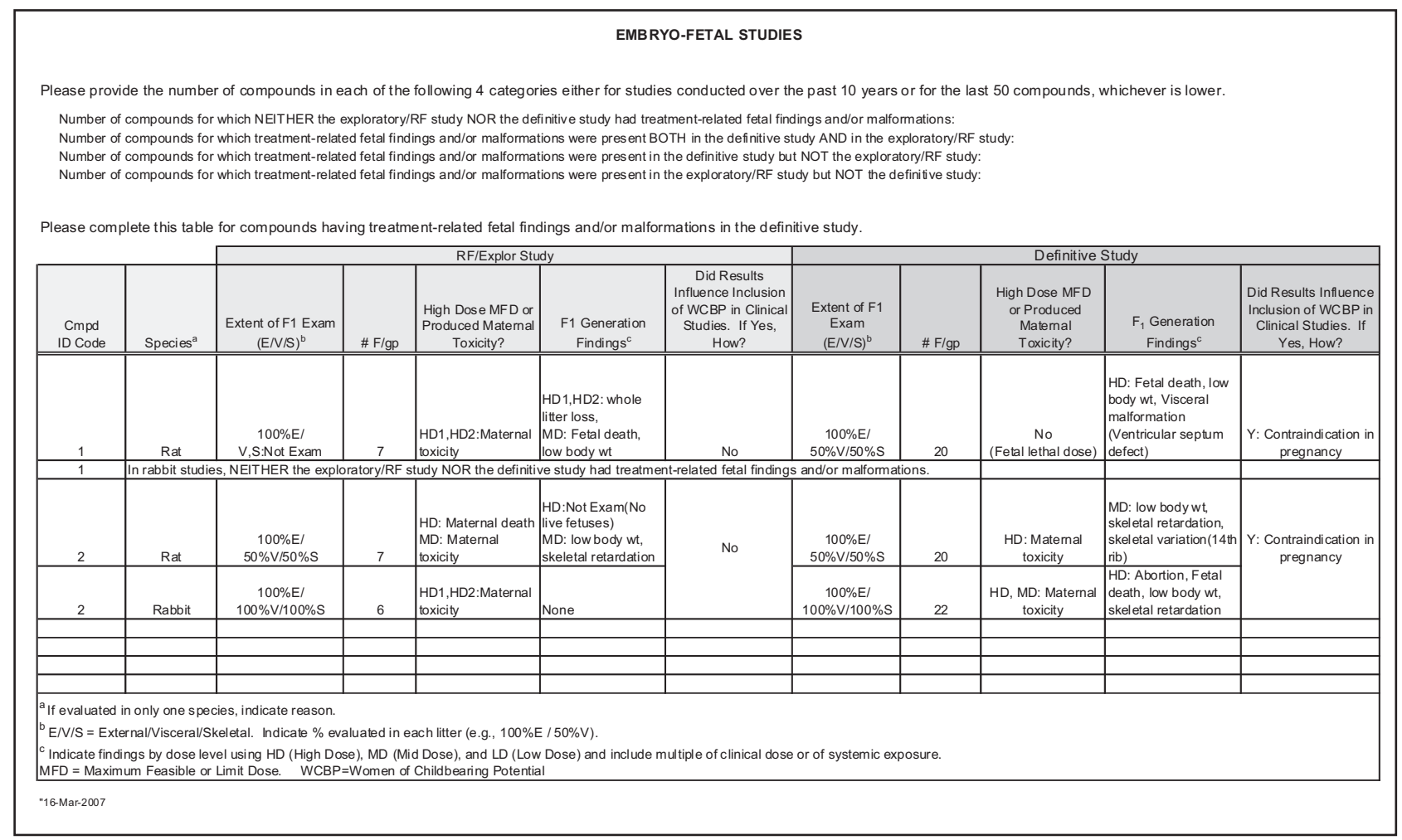

Fig. 1. Example of the answer sheet.

tive value of the preliminary or DRF studies to detect any of the embryo-fetal toxicities by the combination of the rodent and non-rodent studies, (4) influence of the items examined in the preliminary or DRF studies on the predictive value of EFD toxicities. The complete dataset and analysis was also reviewed by the ICH M3 working group.

\section{RESULTS}

\section{Study design of the preliminary or DRF studies}

The methods used in the DRF studies varied between the studies. In almost all the studies, caesarian section was conducted with examination for embryo-fetal death, and fetal body weights were measured. However, the number of dams per group and fetal examination items such as external, visceral and/or skeletal examinations were different between the studies. Regarding the number of the dams per group, 45 out of 108 studies used six dams per group in rodents. The range of the dam numbers per group was 3-13 (minimum - max) in rodents. In non-rodents, 41 out of 85 studies used six dams per group and the range of the dams was 3-8 (minimum -max) (Fig. 2). In the fetal morphological examinations, in about a half of the studies $(44 \%, 48$ out of 108 studies in rodents and $53 \%$, 45 out of 85 studies in non-rodents) were only external examinations done in the DRF studies. In the remaining half of the studies $(51 \%, 55$ out of 108 studies in rodents and $41 \%, 35$ out of 85 studies in non-rodents) full examinations (external, visceral and skeletal) in the DRF studies were done (Fig. 3).

In the results for maternal toxicity, 24 out of 108 compounds showed maternal deaths in the DRF studies, compared with 6 out of 108 compounds in the definitive studies in rodents. In non-rodent studies, 27 out of 85 compounds showed maternal deaths in the DRF studies, compared with 16 out of 85 compounds in the definitive studies.

\section{Predictivity of the preliminary or DRF studies to detect any EFD toxicities in the definitive studies}

As described above, this analysis was conducted with the definitive studies showed some EFD toxicities. If any EFD toxicities including embryo-fetal death, decreased 
N. Okahashi et al.

Table 1. The number of compounds

\begin{tabular}{|c|c|c|c|c|}
\hline \multirow{2}{*}{ Company Code } & \multicolumn{2}{|c|}{ Number of the studies in rodents } & \multirow{2}{*}{$\begin{array}{c}\text { Number of the studies in non-rodents } \\
\text { Rabbits }\end{array}$} & \multirow{2}{*}{ Number of the compounds } \\
\hline & Rats & Mice & & \\
\hline 1 & 2 & 0 & 2 & 2 \\
\hline 2 & 2 & 0 & 1 & 2 \\
\hline 3 & 3 & 0 & 1 & 3 \\
\hline 4 & 1 & 0 & 1 & 2 \\
\hline 5 & 1 & 0 & 3 & 3 \\
\hline 6 & 2 & 0 & 2 & 3 \\
\hline 7 & 0 & 1 & 0 & 1 \\
\hline 8 & 26 & 1 & 23 & 33 \\
\hline 9 & 2 & 0 & 1 & 2 \\
\hline 10 & 9 & 0 & 4 & 9 \\
\hline 11 & 2 & 0 & 2 & 3 \\
\hline 12 & 2 & 0 & 4 & 4 \\
\hline 13 & 9 & 1 & 11 & 14 \\
\hline 14 & $1^{\mathrm{a}}$ & $1^{\mathrm{a}}$ & 0 & $1^{\mathrm{a}}$ \\
\hline 15 & 1 & 0 & 0 & 1 \\
\hline 16 & 1 & 0 & 0 & 1 \\
\hline 17 & 7 & 0 & 2 & 8 \\
\hline 18 & 0 & 0 & 1 & 1 \\
\hline 19 & 6 & 0 & 4 & 7 \\
\hline 20 & 2 & 0 & 1 & 2 \\
\hline 21 & 1 & 0 & 0 & 1 \\
\hline 22 & 13 & 1 & 12 & 21 \\
\hline 23 & 2 & 0 & 2 & 2 \\
\hline 24 & 8 & 0 & 8 & 9 \\
\hline Total & & & 85 & 136 \\
\hline
\end{tabular}

a: The studies in rats and mouse were conducted with the same compound.

fetal body weight, fetal malformation or variation (external, visceral and/or skeletal) and/or retardation of ossification were detected in the DRF studies, the studies were regarded as "positive". The predictive value was calculated by the following formula: (the number of "positive" studies in the DRF studies/ the number of the analyzed studies)*100.

In the results of the analysis, the predictive values of DRF studies for EFD toxicities were 83\% (90 out of 108 studies) and $80 \%$ (68 out of 85 studies) in rodents and non-rodents, respectively (Fig. 4).

\section{Predictivity of the preliminary or DRF studies to detect fetal "malformation" in the definitive studies}

Focusing on the fetal malformations, the same analyses were conducted for the studies in which any malformations (external, visceral and/or skeletal) were detected in the definitive studies. The number of the analyzed studies was 34 for rodents and 21 for non-rodents. In these studies, malformations were detected in 53\% (18 out of 34 studies) and $57 \%$ (12 out of 21 studies) by the DRF studies in rodents and non-rodents, respectively (Fig. 5).

Another analysis was conducted in which the studies were regarded as "positive" when any EFD toxicity including embryo-fetal death, decreased fetal body 
The predictivity of preliminary embryo-fetal development studies

(A)Rodents

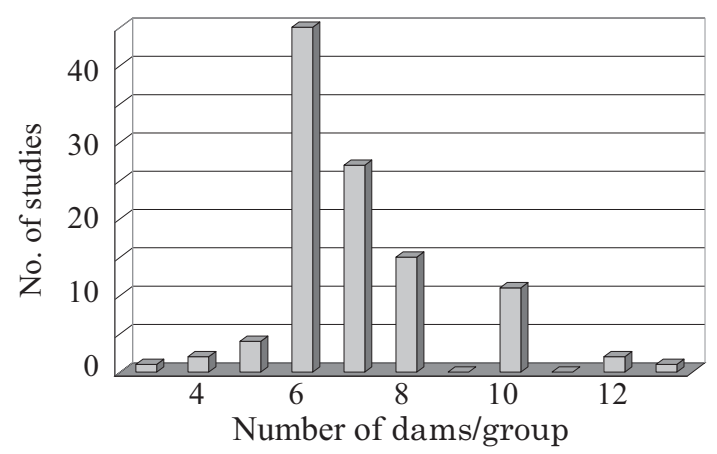

(B)Non-rodents

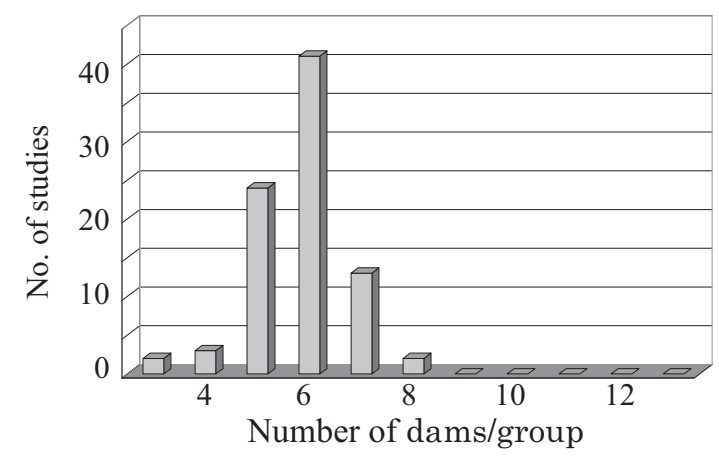

Fig. 2. The number of the studies in each number of the dams per group in the preliminary or DRF studies with rodents (A, Number of analyzed studies was 108.) and non-rodents (B, Number of analyzed studies was 85.).

(A) Rodents

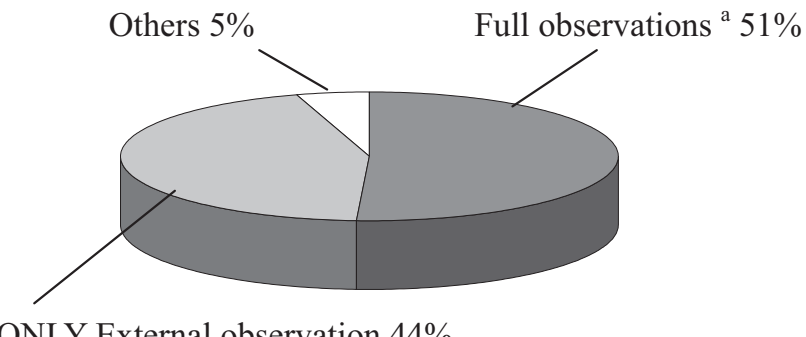

ONLY External observation 44\%
(B) Non-rodents

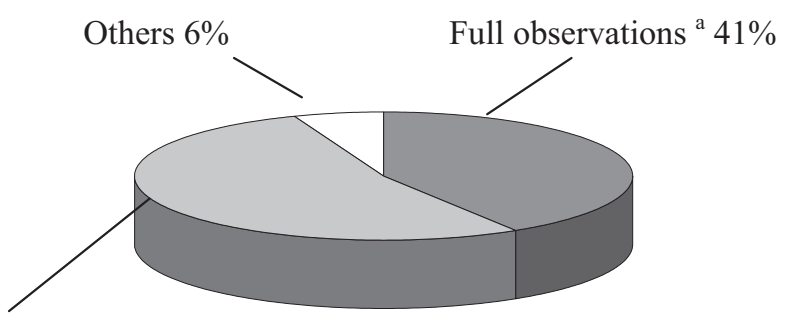

ONLY External observation 53\%

Fig. 3. The percentages of the studies with the observation items for the fetuses in the preliminary or DRF studies with rodents (A, Number of analyzed studies was 108.) and non-rodents (B, Number of analyzed studies was 85.).

a : Full observation means external, visceral and skeletal observations were performed.

weight, fetal malformation or variation (external, visceral and/or skeletal) and/or fetal retardation of ossification was detected in the DRF studies. In the results of the analysis, 91\% (31 out of 34 studies) in rodents and 100\% (21 out of 21 studies) in non-rodents were judged "positive" (Fig. 6).

Only 3 studies in rodents exhibited no EFD toxicity in the DRF studies because of a low incidence of malformations (2 studies; they had no effects on fetal death or body weights in both DRF and definitive studies.) or the lack of the appropriate examination (1 study; visceral and skeletal examinations were not conducted and only visceral malformation was observed in the definitive study.) in the DRF studies.

\section{Predictivity of the preliminary or DRF studies to detect any EFD toxicity or fetal "malformation" in the definitive studies by the combination of the rodent and non-rodent studies}

When the effects of both the rodent and non-rodent studies were evaluated together, 56 compounds had both rodent and non-rodent data sets, that is, these 56 compounds showed EFD toxicity in both rodents and nonrodents in the definitive studies.

First, the analysis was conducted with "EFD toxicants", in which any EFD toxicity including embryofetal death, decreased fetal body weight, fetal malformation or variation (external, visceral and/or skeletal) and/or retardation of fetal ossification were observed in both the rodent or non-rodent definitive studies. The predictive value was calculated by the following formula: (the number of "positive" compounds in the DRF studies/ the number of the analyzed compounds)*100 In this formu- 
(A)Rodents

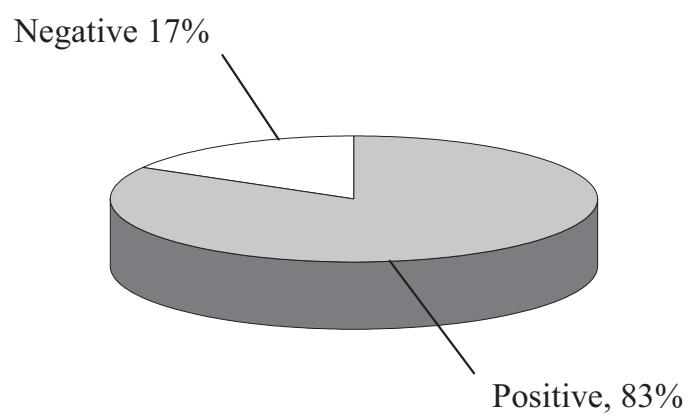

(B)Non-rodents

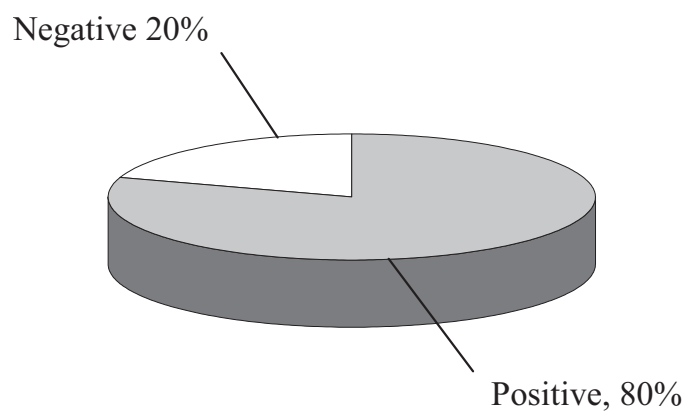

Fig. 4. The percentages of "positive" or "negative" studies in the preliminary or DRF studies for any EFD effects in rodents (A, Number of analyzed studies was 108.) and non-rodents (B, Number of analyzed studies was 85).

Positive: Effects on the any EFD were detected in the preliminary or DRF studies.

Negative: No effects on the EFD were observed in the preliminary or DRF studies.

(A)Rodents

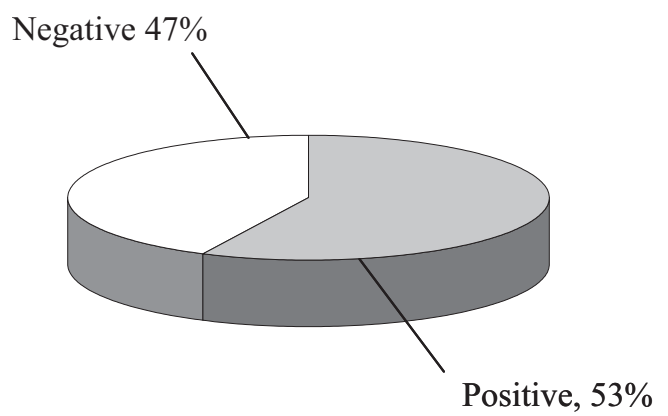

(B)Non-rodents

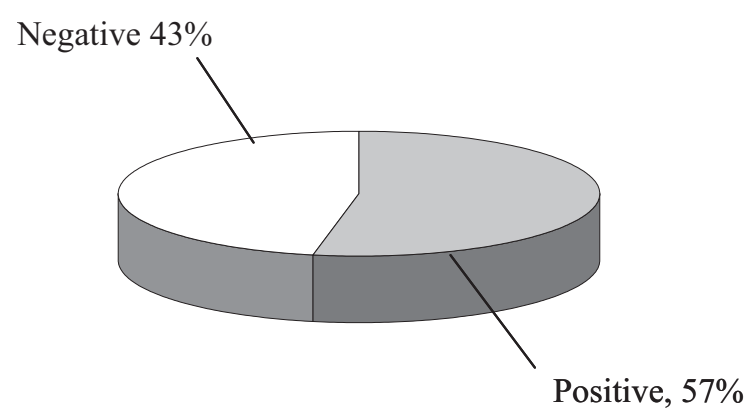

Fig. 5. The percentages of "positive" or "negative" studies in the preliminary or DRF studies for any fetal malformations (external, visceral and/or skeletal) in rodents (A, Number of analyzed studies was 34.) and non-rodents (B, Number of analyzed studies was 21.).

Positive: Any malformations were detected in the preliminary or DRF studies.

Negative: No malformations were observed in the preliminary or DRF studies.

la, "positive" meant any EFD effects were detected in both or either rodent and non-rodent preliminary or DRF studies. The combination predictive value for "EFD toxicants" was $96 \%$ (54 out of 56 compounds).

Next, focusing on the fetal malformations, the same analyses were conducted for the "teratogens" in which any malformations (external, visceral and/or skeletal) were detected in the definitive studies on both or either rodents or non-rodents. The combination predictive value for the teratogens which had both rodent and non-rodent data sets was $100 \%$ (23 out of 23 compounds) (Table 2).

\section{Influence of the examination items in the predictivity of the preliminary or DRF studies}

To analyze the influence of the items examined, the predictive value was calculated assuming that fetal skeletal and visceral examinations were not conducted, that is, the EFD effects were evaluated only by embryo-fetal death, decreased fetal body weight and/or external examinations.

There were 54 analyzed compounds for which full examinations (external, visceral and skeletal examination) were conducted in both rodent and non-rodent DRF studies. The analysis was conducted in the same manner as the combination analysis described above. The combination 
The predictivity of preliminary embryo-fetal development studies

(A)Rodents

Negative $9 \%$

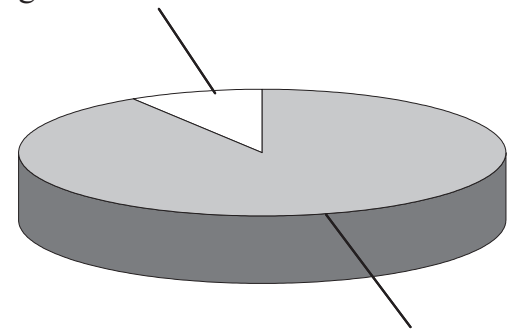

Positive, $91 \%$
(B)Non-rodents

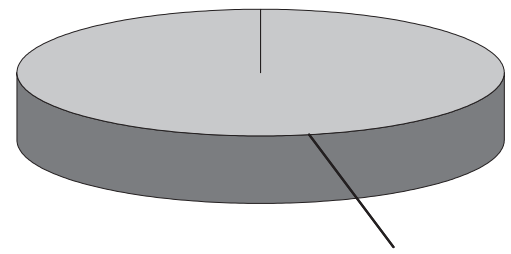

Positive, $100 \%$

Fig. 6. The percentages of "positive" or "negative" studies in the preliminary or DRF studies for any fetal malformations (external, visceral and/or skeletal) in rodents (A, Number of analyzed studies was 34) and non-rodents (B, Number of analyzed studies was 21.).

Positive: Effects on EFD were detected in the preliminary or DRF studies.

Negative: No effects on EFD were observed in the preliminary or DRF studies.

Table 2. The Predictivity of preliminary or DRF studies by the combination of rodent and non-rodent studies

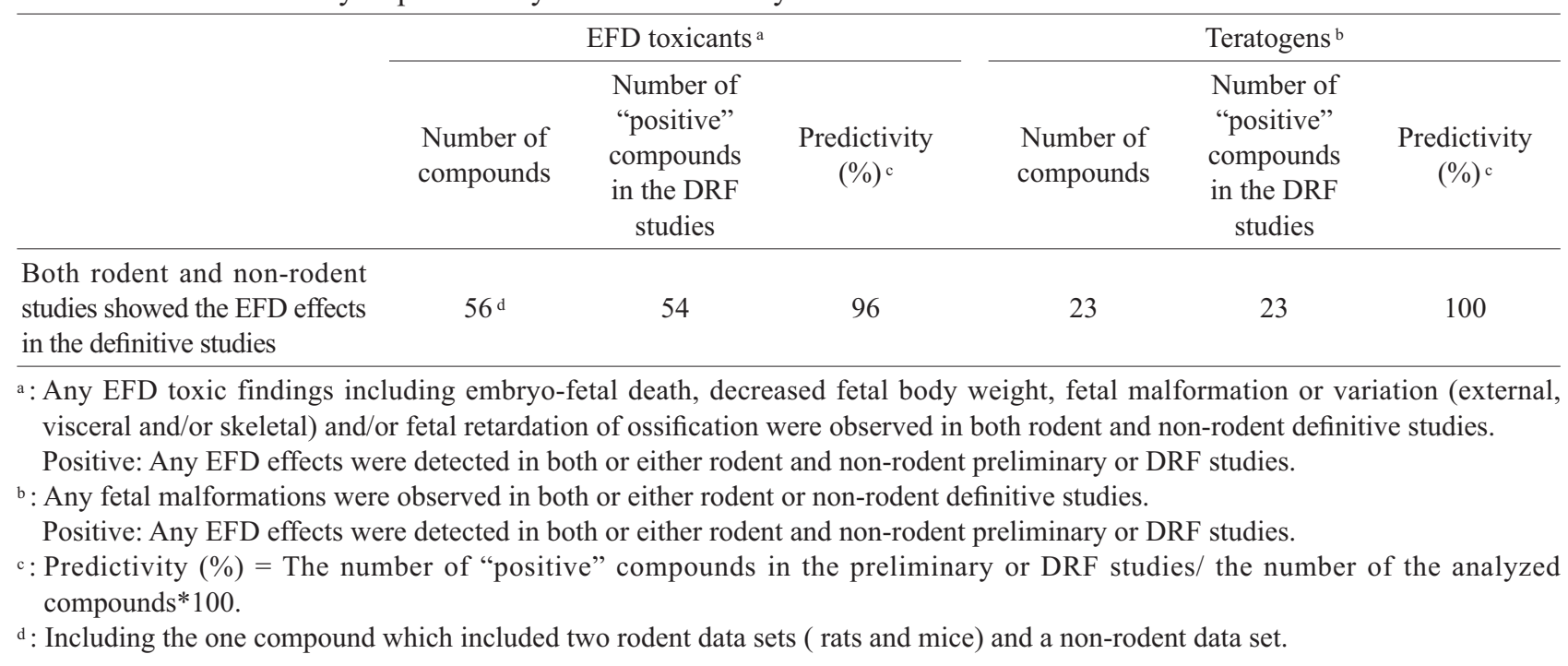

predictive value was $93 \%$ (50 out of 54 compounds) and $87 \%$ (47 out of 54 compounds) for the "EFD toxicants", with full examination and without visceral and skeletal examinations results, respectively (Table 3 ). That is, the EFD effects could not be detected in 3 compounds without visceral and skeletal examinations results; two compounds showed only skeletal variation as a fetal effect in the DRF studies in rodents, and one compound showed only skeletal retardation as a fetal effect in the DRF studies in non-rodents.

Focusing on the fetal malformations, the combination predictive value was $95 \%$ (20 out of 21 compounds) for the "teratogens" in both situations (Table 3). Differences in the predictivity between the values including and excluding fetal visceral or skeletal examinations results were not evident.

In addition, another analysis was conducted to estimate the influence of the examination items. For the compounds for which full observations in the DRF studies were performed and teratogenicity in the definitive studies were noted, the results in the DRF studies and the definitive studies were compared (Tables 4 and 5). If external observation, fetal death and low fetal body weight are used as markers, only 1 of 17 compounds could not be 
Table 3. Predictivity of preliminary or DRF studies by the combination of rodent and non-rodent studies In the case in which the embryo-fetal effects were evaluated only by embryo-fetal death and/or decreased fetal body weight

\begin{tabular}{|c|c|c|c|c|c|c|}
\hline & \multicolumn{3}{|c|}{ EFD toxicants a } & \multicolumn{3}{|c|}{ Teratogens $^{b}$} \\
\hline & $\begin{array}{l}\text { Number of } \\
\text { compounds }\end{array}$ & $\begin{array}{l}\text { Number of } \\
\text { the "positive" } \\
\text { compounds }\end{array}$ & $\begin{array}{c}\text { Predictivity } \\
(\%)^{c}\end{array}$ & $\begin{array}{l}\text { Number of } \\
\text { compounds }\end{array}$ & $\begin{array}{l}\text { Number of } \\
\text { "positive" } \\
\text { compounds }\end{array}$ & $\begin{array}{c}\text { Predictivity } \\
(\%)^{c}\end{array}$ \\
\hline Evaluation by full examination & 54 & 50 & 93 & 21 & 20 & 95 \\
\hline $\begin{array}{l}\text { Evaluation only by embryo- } \\
\text { fetal death, decreased fetal } \\
\text { body weight, and/or external } \\
\text { examination }\end{array}$ & 54 & 47 & 87 & 21 & 20 & 95 \\
\hline
\end{tabular}

a : Any EFD toxic findings including embryo-fetal death, decreased fetal body weight, fetal malformation or variation (external, visceral and/or skeletal) and/or fetal retardation of ossification were observed in both rodent or non-rodent definitive studies.

$\mathrm{b}$ : Any fetal malformations were observed in both or either rodent or non-rodent definitive studies.

c: Predictivity $(\%)=$ The number of "positive" compounds in the preliminary or DRF studies/ the number of compounds analyzed $* 100$.

detected the EFD toxicity in the DRF study in rodents. The compound showed no effects on skeletal and visceral observations so it would not be positive even if skeletal and visceral examinations were performed. In nonrodents, no compound ( 0 of 10 compounds) was missed.

\section{DISCUSSION}

In this survey among member companies of the JPMA, we assessed the design of the DRF study in the rodents and non-rodents and their predictivity by comparing with the results of the definitive EFD toxicity studies conducted to GLP.

As for the study design in the DRF studies, six dams per group were most common and about a half of the studies were conducted with only external examination for fetal morphology in both the rodents and non-rodents. These results may indicate that the DRF studies are simply designed to set the dose range for the definitive studies rather to detect the detailed information about the EFD toxicity.

The number of the DRF studies showing maternal death was higher than that in the definitive studies in both rodents and non-rodents. These results may suggest that the maximum dose tested was relatively high in the DRF studies compared to the definitive studies. This is probably because the DRF studies are conducted with limited information.

Under these study conditions, the predictive values of the DRF studies to detect any EFD toxicities in the definitive studies was more than $80 \%$ in both rodent and nonrodent studies. There was no obvious difference between species for the predictivity.

For the teratogens which showed malformations in the definitive studies, the predictive value of the DRF study in which the malformations were detected was only about $50 \%$ in both the rodents and non-rodents. This may indicate that negative teratogenic findings in the DRF study do not preclude teratogenic findings in the definitive study where larger numbers of animals and more refined dose levels are used. However, the predictive value for the teratogenic findings in the definitive studies was higher $(91 \%$ in rodents and $100 \%$ in non-rodents) when any developmental effects including fetal death and/or low fetal body weight in the DRF studies were used as potential predictive markers for adverse fetal effects. Therefore, the evaluation including the endpoints of fetal body weight and embryo-fetal death was considered to be important to evaluate potential teratogenicity. There were 3 compounds which showed no EFD effects in the DRF study but showed malformation in the definitive studies. The DRF studies for two compounds were conducted in the small scale as usual and the incidences of malformation in the definitive studies were very low, and one DRF study didn't conduct the full observation, thus, the DRF studies were likely underpowered to detect the effects.

The combination analysis with rodent and non-rodent results showed that the predictive values for the combination was higher than that for each result by a complementary effect and the values were $96 \%$ for "EFD toxicants" and $100 \%$ for teratogens. This result suggests that the predictivity becomes very high if a compound is studied in the DRF in 2 species.

The analysis of the influence of the observation items 
The predictivity of preliminary embryo-fetal development studies

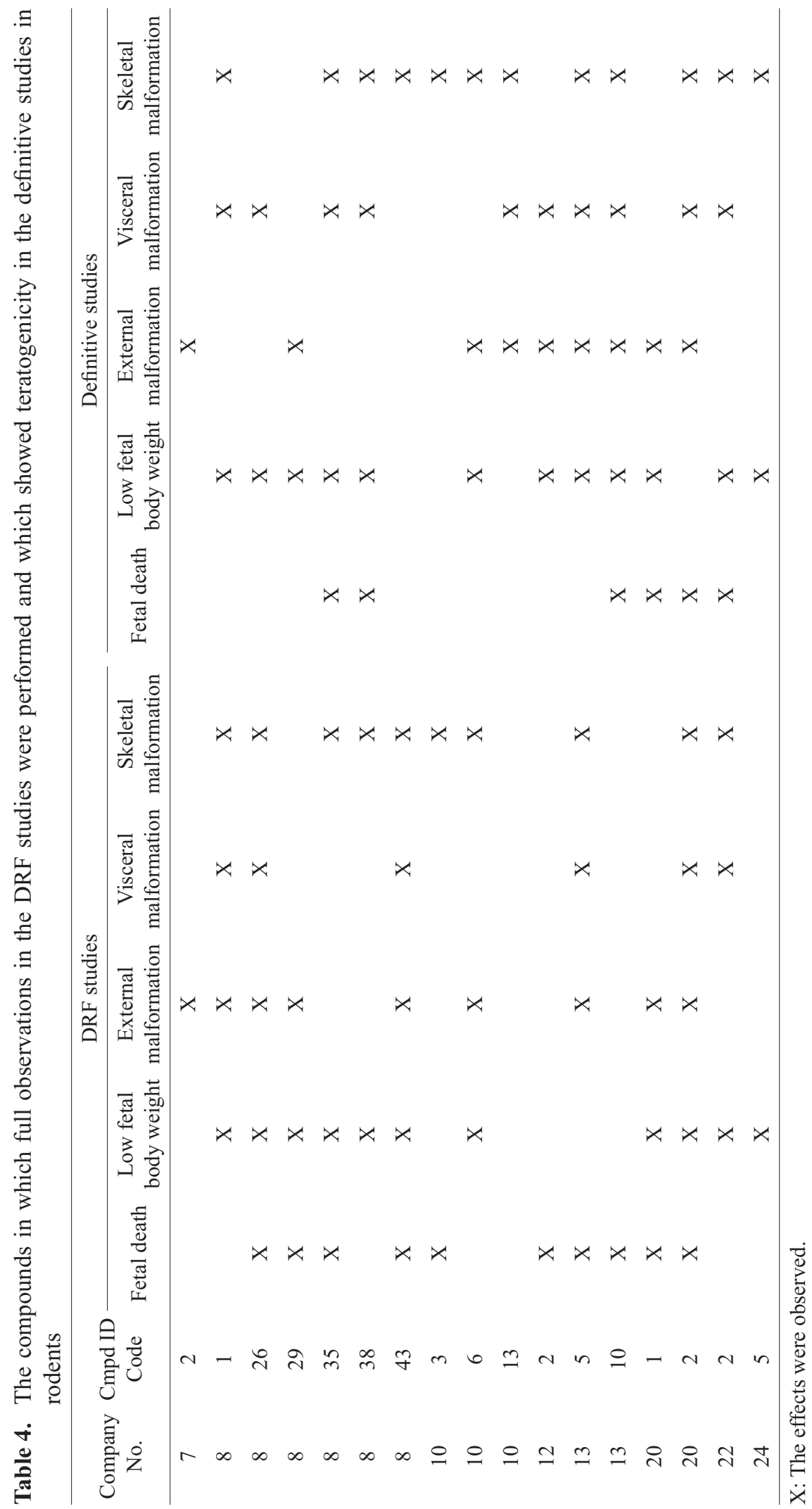


N. Okahashi et al.

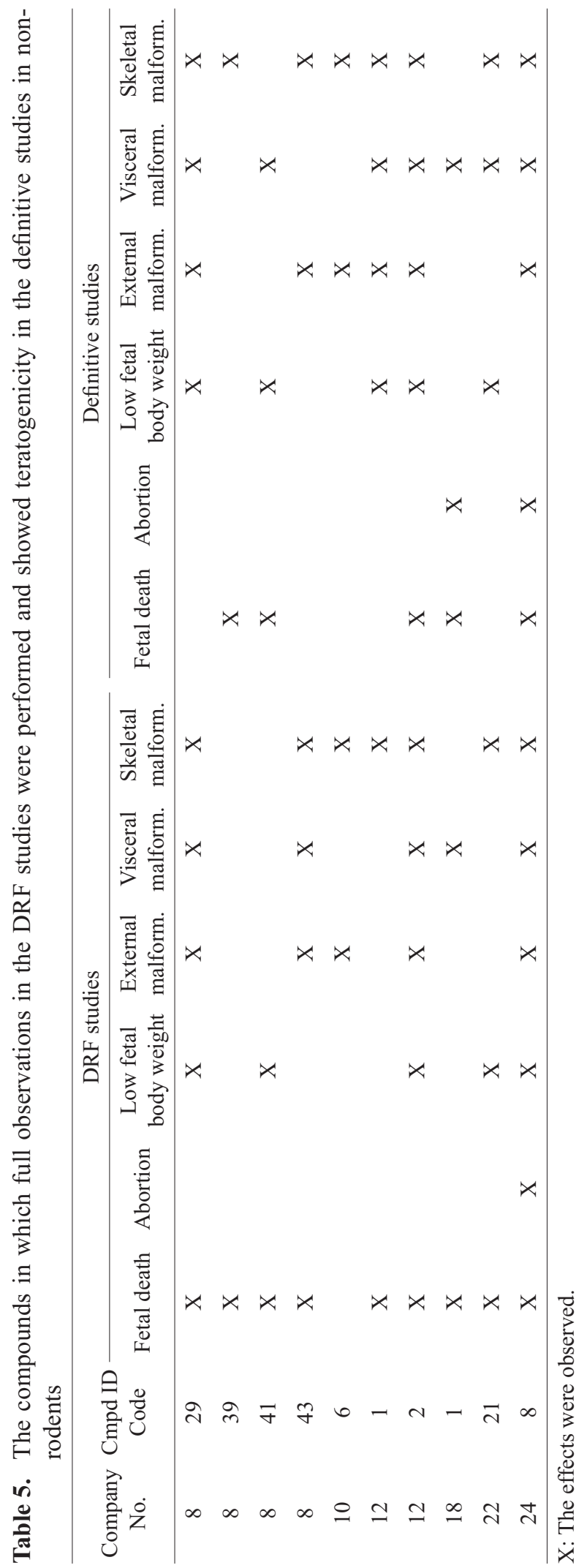

Vol. 35 No. 1 
The predictivity of preliminary embryo-fetal development studies

revealed that the differences in the predictive value between the limited examinations (i.e., external observation only) and full examinations were not significant for teratogens and EFD toxicants. Moreover, there were no compounds which were detected only by visceral examination. It is well known that many EFD toxicants represent a continuum of increasing toxicity, with low doses producing growth retardation (low fetal body weight) and increasing doses producing malformations and then lethality (Rogers and Kavlock, 2007). Consequently, it is reasonable that the end points of fetal body weight and embryo-fetal death are useful markers to detect the embryo-fetal toxicants.

In conclusion, the results of this survey showed that a pair of the DRF studies in the rodent and non-rodent species are considered to be able to provide critical information which could be comparable to those from the definitive study if adequate dose levels, numbers of dams, appropriate observation items such as fetal survival, body weight and external examination are used. It should be noted that fetal lethality and decreased fetal body weights should be regarded not only as the signal of the developmental retardation but also as a signal that may herald teratogenic potential.

\section{Limitation of interpretation}

There may be limitations to the generalization of the interpretation of this analysis because: 1) the numbers of the compounds and the companies are limited; 2) the interpretation was based on the hypothesis that there is no difference in methodology between the DRF study and definitive study except for dosage, number of dams and examination items; however, there could be unknown differences such as toxicokinetics in the dams. However, such differences are considered small because the companies have used these data to make their internal decisions on dose selection for subsequent studies. On the other hand, this survey focused on the sensitivity of the DRF study and therefore, the specificity of DRF studies has not been elucidated through this survey. The proposed setting of supporting early clinical development, however, sensitivity is considered to be more important for the purpose of early identification of the potential EFD toxicity. It is concluded from the above analysis that the DRF studies are sufficiently useful to support early clinical trails when strategies to minimize pregnancy rates are incorporated into the clinical design.

\section{ACKNOWLEDGMENTS}

We acknowledge all of the researchers of the JPMA members replying willingly to the questions.

\section{REFERENCES}

ICH harmonized tripartite guideline (2000): Harmonized Tripartite Guideline: Maintenance of the ICH guideline on Non-clinical Safety studies for the Conduct of Human Clinical Trials for Pharmaceuticals M3 (R1) (available at http://www.pmda.go.jp/ $\mathrm{ich} / \mathrm{m} / \mathrm{m} 3 \mathrm{r} 1 \quad 00 \quad 12$ 27e.pdf).

ICH harmonized tripartite guideline (2005): Detection of toxicity to reproduction for medicinal products $\&$ toxicity to male fertility S5 (R2) (available at http://www.pmda.go.jp/ich/s/s5r2_00_12_ 27e.pdf).

Rogers, J.M. and Kavlock, R.J. (2007): Developmental Toxicology. In Casarett and Doull's toxicology - The basic science of poisons 6th ed. (Klaassen, C.D. 7th ed.), 415-451, McGraw-Hill, New York. 